\title{
EXPLICIT INTERPOLATION BOUNDS BETWEEN HARDY SPACE AND $L^{2}$
}

\author{
H.-Q. BUI and R. S. LAUGESEN ${ }^{凶}$ \\ (Received 27 June 2012; accepted 5 March 2013; first published online 18 July 2013) \\ Communicated by A. Hassell
}

\begin{abstract}
Every bounded linear operator that maps $H^{1}$ to $L^{1}$ and $L^{2}$ to $L^{2}$ is bounded from $L^{p}$ to $L^{p}$ for each $p \in(1,2)$, by a famous interpolation result of Fefferman and Stein. We prove $L^{p}$-norm bounds that grow like $O(1 /(p-1))$ as $p \downarrow 1$. This growth rate is optimal, and improves significantly on the previously known exponential bound $O\left(2^{1 /(p-1)}\right)$. For $p \in(2, \infty)$, we prove explicit $L^{p}$ estimates on each bounded linear operator mapping $L^{\infty}$ to bounded mean oscillation $(B M O)$ and $L^{2}$ to $L^{2}$. This $B M O$ interpolation result implies the $H^{1}$ result above, by duality. In addition, we obtain stronger results by working with dyadic $H^{1}$ and dyadic $B M O$. The proofs proceed by complex interpolation, after we develop an optimal dyadic 'good lambda' inequality for the dyadic $\sharp$-maximal operator.
\end{abstract}

2010 Mathematics subject classification: primary 42B30; secondary 46B70.

Keywords and phrases: BMO, bounded mean oscillation, interpolation of operators.

\section{Introduction}

A central interpolation result in harmonic analysis says a linear operator that is bounded on $L^{2}$ and on the Hardy space $H^{1}$ must extend to a bounded operator on $L^{p}$, whenever $1<p<2$. The size of the $L^{p}$-norm of the operator can matter a great deal. For example, a linear operator $T$ is invertible on $L^{p}$ if it lies within distance 1 of the identity map, meaning $\|I-T\|_{L^{p} \rightarrow L^{p}}<1$. Thus if we had explicit interpolation bounds on $I-T$, then we would know how close $T$ must lie to the identity on $H^{1}$ and $L^{2}$ in order to ensure that the Neumann series for $(I-T)^{-1}$ converges on $L^{p}$. Such questions arose recently in our work on wavelet frame operators [1].

We begin by interpolating between the dual spaces, which are $L^{2}$ and bounded mean oscillation $(B M O)$. The fundamental result of Fefferman and Stein [3] assumes $T$ : $L^{2} \rightarrow L^{2}$ and $T: L^{\infty} \rightarrow B M O$, and deduces explicit $L^{p}$ bounds for $2<p<\infty$. We will

This paper was partially supported by a grant from the Simons Foundation (\#204296 to Richard Laugesen).

(C) 2013 Australian Mathematical Publishing Association Inc. 1446-7887/2013 \$16.00 
prove a version of this interpolation theorem in which the space $B M O$ is replaced by the dyadic space $d B M O$ (defined in Section 2), with explicit constants that improve considerably on those known previously. The constant appearing in the theorem is

$$
K(p)=\left[(1+1 / p)^{p+2}(p+1)^{1 / p}\right] p .
$$

Write $L^{p}=L^{p}\left(\mathbb{R}^{n}\right), n \geq 1$.

Theorem 1 ( $L^{2}$ and dBMO interpolation). Suppose $T$ is a linear operator defined on $L^{2}+L^{\infty}$ that takes values in the space of complex valued measurable functions on $\mathbb{R}^{n}$.

If $T$ is strong type $\left(L^{2}, L^{2}\right)$ and strong type $\left(L^{\infty}, d B M O\right)$, then $T$ is strong type $\left(L^{p}, L^{p}\right)$ whenever $2 \leq p<\infty$, with

$$
\|T\|_{L^{p} \rightarrow L^{p}} \leq 2^{n+4 / p} K(p)\|T\|_{L^{2} \rightarrow L^{2}}^{2 / p}\|T\|_{L^{\infty} \rightarrow d B M O}^{1-2 / p} .
$$

The analogous result holds (of course) when $d B M O$ is replaced by the smaller space $B M O$.

We have found in the literature three distinct lines of proof for the $B M O$ version of this theorem. First, Fefferman and Stein use complex interpolation along with the $\sharp$-maximal operator and, in essence, a good lambda inequality [3, Corollary 2]. Their work yields a constant of order $2^{(n+1) p}$. Second, the approach presented in Stein's monograph uses complex interpolation along with estimates of the $L^{p}$-norm in terms of the $\sharp$-maximal norm, via the grand maximal function [8, pages 148, 175-177]. This approach does not appear capable of providing an explicit constant. Third, Grafakos uses real interpolation along with the dyadic $\sharp$-maximal operator and a good lambda inequality [5, Theorem 7.4.7]. This approach gives a constant of order $2^{p}$.

Thus $2^{p}$ or not $2^{p}$, that is the question. We resolve it by modifying the first line of proof: we use complex interpolation with the dyadic $\sharp$-maximal operator and an optimized application of a good lambda inequality (see Proposition 9). The constant then improves dramatically. Rather than growing like $2^{p}$ it grows only linearly. Indeed, $K(p)=O(p)$ with

$$
\frac{81 \sqrt{3}}{16} \geq \frac{K(p)}{p} \geq e \quad \text { for } 2 \leq p<\infty,
$$

because $K(p) / p$ is decreasing (by elementary estimates).

Linear growth of the constant is the best possible rate, since the $L^{p}$-norm of the Hilbert transform equals $\cot (\pi / 2 p) \sim(2 / \pi) p$ as $p \rightarrow \infty$, as Pichorides showed [7], and similarly for the Riesz transform in higher dimensions [6, Theorem 1.9.1].

We do not know whether the exponential growth $2^{n}$ of the constant in Theorem 1 is best possible with respect to dimension. The factor of $2^{n}$ arises in the proof of Lemma 8, when the sides of a cube are doubled; perhaps an alternative approach could be found?

REMARK 2. Fefferman and Stein proved a more general interpolation theorem, for an analytic family of operators rather than the fixed operator $T$. Our methods improve the constant there too. 
Interpolation between $\boldsymbol{H}^{\mathbf{1}}$ and $\boldsymbol{L}^{\mathbf{2}}$. Theorem 1 and the duality relation $\left(H^{1}\right)^{*}=B M O$ together yield interpolation between $L^{2}$ and the Hardy space $H^{1}\left(\mathbb{R}^{n}\right)$. On the Hardy space we use the atomic norm in terms of $L^{\infty}$-atoms, in order to respect duality with the $B M O$-norm.

Corollary 3 ( $H^{1}$ and $L^{2}$ interpolation). Suppose $T$ is a linear operator defined on $H^{1}+L^{2}$ that takes values in the space of complex-valued measurable functions on $\mathbb{R}^{n}$.

If $T$ is strong type $\left(H^{1}, L^{1}\right)$ and strong type $\left(L^{2}, L^{2}\right)$, then $T$ is strong type $\left(L^{p}, L^{p}\right)$ whenever $1<p \leq 2$, with

$$
\|T\|_{L^{p} \rightarrow L^{p}} \leq 2^{n+3-2 / p} K\left(\frac{p}{p-1}\right)\|T\|_{H^{1} \rightarrow L^{1}}^{(2 / p)-1}\|T\|_{L^{2} \rightarrow L^{2}}^{2-(2 / p)} .
$$

Note that the constant $K(p /(p-1))$ has order of growth $O(1 /(p-1))$ as $p \downarrow 1$. This rate is best possible, by the example of the Hilbert transform.

Interpolation between dyadic $\boldsymbol{H}^{\mathbf{1}}$ and $\boldsymbol{L}^{\mathbf{2}}$. Write $H_{d}^{1}\left(\mathbb{R}^{n}\right)$ for the dyadic Hardy space, which is defined in terms of $L^{\infty}$-atoms supported in dyadic cubes. The dual space $\left(H_{d}^{1}\right)^{*}$ is known to be identified with $d B M O$. Using this duality, one may easily adapt the proof of Corollary 3 to obtain an analogue of the corollary with $H^{1}$ replaced by the smaller space $H_{d}^{1}$.

The next two sections develop properties of the dyadic $\sharp$-maximal operator. Then in Section 4 we prove Theorem 1 and Corollary 3.

\section{Pointwise properties of the dyadic $\sharp$-maximal operator}

For $f \in L_{\text {loc }}^{1}$ we recall the dyadic maximal operator

$$
\left(M_{d} f\right)(x)=\sup \left\{\frac{1}{|Q|} \int_{Q}|f(y)| d y: Q \text { is a dyadic cube in } \mathbb{R}^{n} \text { containing } x\right\},
$$

and the dyadic $\sharp$-maximal operator of Fefferman and Stein

$$
\left(M_{d}^{\sharp} f\right)(x)=\sup \left\{\frac{1}{|Q|} \int_{Q}\left|f(y)-f_{Q}\right| d y: Q \text { is a dyadic cube in } \mathbb{R}^{n} \text { containing } x\right\},
$$

where

$$
f_{Q}=\frac{1}{|Q|} \int_{Q} f(y) d y
$$

denotes the average value of $f$ over $Q$. The usual maximal operator $M$ and $\sharp$-maximal operator $M^{\sharp}$ are defined similarly, using all cubes instead of just dyadic cubes.

Define the $B M O$ and $d B M O$ seminorms by

$$
\|f\|_{B M O}=\left\|M^{\sharp} f\right\|_{L^{\infty}}, \quad\|f\|_{d B M O}=\left\|M_{d}^{\sharp} f\right\|_{L^{\infty}} .
$$


The Banach space $B M O$ consists of functions (modulo additive constants) for which the $B M O$ seminorm is finite, while $d B M O$ consists of functions (modulo additive constants in each quadrant of $\mathbb{R}^{n}$ ) for which the $d B M O$ seminorm is finite. Clearly the $d B M O$ norm is smaller in general, and so

$$
B M O \hookrightarrow d B M O .
$$

For more information on dyadic $B M O$, see, for example, the paper of Garnett and Jones [4].

The dyadic $\sharp$-maximal operator is dominated pointwise by the dyadic maximal operator, with $M_{d}^{\sharp} f \leq 2 M_{d} f$. Further pointwise properties are developed in what follows.

Lemma 4 (Measurability). If $f \in L_{\mathrm{loc}}^{1}$ then $M_{d}^{\sharp} f$ is measurable.

Proof. Fix the function $f$ and rewrite the definition of the dyadic $\sharp$-maximal operator as

$$
M_{d}^{\sharp} f(x)=\sup \left\{O_{Q}(x): Q \text { is a dyadic cube in } \mathbb{R}^{n}\right\},
$$

where

$$
O_{Q}(x)=1_{Q}(x) \frac{1}{|Q|} \int_{Q}\left|f(y)-f_{Q}\right| d y .
$$

(Here $O$ indicates 'oscillation'.) This formula expresses $M_{d}^{\sharp} f$ as the supremum of a countable family of measurable functions.

Lemma 5 (Supremum attained). If $f \in L^{p}, 1 \leq p<\infty$, and $x$ is a Lebesgue point for $f$, then there exists a dyadic cube $Q(x)$ containing $x$ such that

$$
M_{d}^{\sharp} f(x)=\frac{1}{|Q(x)|} \int_{Q(x)}\left|f(y)-f_{Q(x)}\right| d y .
$$

Thus the supremum in the definition of the dyadic $\sharp$-maximal operator is attained, for almost every $x$.

Proof. If $M_{d}^{\sharp} f(x)=0$ then any dyadic cube containing $x$ will do. For definiteness, we choose $Q(x)$ to be the unit dyadic cube containing $x$.

Assume that $M_{d}^{\sharp} f(x)>0$. First we show that we need not consider cubes with large volume. Indeed,

$$
\begin{aligned}
\frac{1}{|Q|} \int_{Q}\left|f(y)-f_{Q}\right| d y & \leq 2 \frac{1}{|Q|} \int_{Q}|f(y)| d y \\
& \leq 2\left(\frac{1}{|Q|} \int_{Q}|f(y)|^{p} d y\right)^{1 / p} \\
& \leq 2 \frac{1}{|Q|^{1 / p}}\|f\|_{L^{p}} \rightarrow 0
\end{aligned}
$$

as $|Q| \rightarrow \infty$ (using here that $p<\infty$ ). 
Second, we need not consider cubes with small volume, since if $Q$ contains $x$ then

$$
\lim _{|Q| \rightarrow 0} \frac{1}{|Q|} \int_{Q}\left|f(y)-f_{Q}\right| d y=0
$$

because $x$ is a Lebesgue point.

Combining these two observations, we see that only finitely many dyadic cubes need to be considered when evaluating the supremum for $M_{d}^{\sharp} f(x)$. Thus the supremum is attained for some cube, which we call $Q(x)$.

The proof above leads to a locally uniform upper bound on the size of the cube $Q(x)$.

Lemma 6 (Upper bound on cube size). Suppose $f \in L^{p}, 1 \leq p<\infty$, and let $\widetilde{Q}$ be a dyadic cube. Then $|Q(x)|$ is bounded, as a function on Lebesgue points $x \in \widetilde{Q}$.

Proof. Suppose that $\int_{Q}\left|f(y)-f_{Q}\right| d y>0$ for some dyadic cube $Q$ containing $\widetilde{Q}$. Then for each Lebesgue point $x \in \widetilde{Q}$,

$$
\begin{aligned}
0<\frac{1}{|Q|} \int_{Q}\left|f(y)-f_{Q}\right| d y \leq M_{d}^{\sharp} f(x) & =\frac{1}{|Q(x)|} \int_{Q(x)}\left|f(y)-f_{Q(x)}\right| d y \\
& \leq 2 \frac{1}{|Q(x)|^{1 / p}}\|f\|_{L^{p}}
\end{aligned}
$$

by estimate (1) applied to $Q(x)$. Rearranging this inequality gives a uniform upper bound on $Q(x)$.

Suppose on the other hand that $\int_{Q}\left|f(y)-f_{Q}\right| d y=0$ for each dyadic cube $Q$ containing $\widetilde{Q}$. Then in particular $f$ is constant almost everywhere on $\widetilde{Q}$, and so $\int_{Q}\left|f(y)-f_{Q}\right| d y=0$ for each dyadic cube $Q$ contained in $\widetilde{Q}$. We have supposed that the same equality holds when $Q$ contains $\widetilde{Q}$, and so necessarily $M_{d}^{\sharp} f(x)=0$ for each $x \in \widetilde{Q}$. Hence each $Q(x)$ is a unit cube by construction as above, and so $|Q(x)|=1$, which completes the proof.

Next we evaluate the dyadic $\sharp$-maximal operator at $f$ in terms of a linear integral operator that depends on $f$. This representation is a dyadic version of one due to Fefferman and Stein [3], and is central to the proof of Theorem 1.

We will treat the issue of measurability carefully, in the next result, since it seems not to be addressed in the literature. Fortunately, measurability is easier to establish for the dyadic case than for the original $\sharp$-maximal operator that uses all cubes.

Write $Q_{d}$ for the collection of all dyadic cubes.

Proposition 7 (Linearization and measurability). If $f \in L^{p}, 1 \leq p<\infty$, then there exist functions $Q: \mathbb{R}^{n} \rightarrow Q_{d}$ and $\kappa: \mathbb{R}^{n} \times \mathbb{R}^{n} \rightarrow \mathbb{C}$ such that the set $\{(x, y): y \in Q(x)\}$ is measurable, $\kappa$ is measurable, $|\kappa| \leq 1$, and

$$
M_{d}^{\sharp} f(x)=\frac{1}{|Q(x)|} \int_{Q(x)}\left(f(y)-f_{Q(x)}\right) \kappa(x, y) d y \quad \text { a.e. }
$$


The right side of (2) is related to the ' $U$ ' operator of Fefferman and Stein [3], although they choose $Q(x)$ from the collection of all cubes, whereas we restrict to dyadic cubes. One may interpret (2) as representing $M_{d}^{\sharp}$ as a linear integral operator acting on $f$, although that is incorrect in practice, because the family of cubes $Q$ and the kernel $\kappa$ in (2) depend upon the function $f$.

Proof. Begin by listing the family of dyadic cubes in some order, as $\left\{Q_{j}\right\}_{j=1}^{\infty}$.

Let $x \in \mathbb{R}^{n}$. If $M_{d}^{\sharp} f(x)=0$ then $f$ is constant (and hence zero) on the entire quadrant of $\mathbb{R}^{n}$ containing $x$. Choose $j(x) \geq 1$ to be the $j$-value such that $Q_{j}$ is the unit dyadic cube containing $x$. Do likewise for each point $x$ in the quadrant.

In quadrants where $f$ is not constant we proceed as follows. Suppose $x$ is a Lebesgue point for $f$. Note that $M_{d}^{\sharp} f(x)>0$ because $f$ is not constant on the quadrant. Let $j(x) \geq 1$ be the smallest $j$-value such that $O_{Q_{j}}(x)=M_{d}^{\sharp} f(x)$; such a $j$-value exists by Lemma 5, with $x \in Q_{j(x)}$. The integer-valued function $j(x)$ is measurable, since the functions $M_{d}^{\sharp}$ and $O_{Q_{1}}, O_{Q_{2}}, O_{Q_{3}}, \ldots$ are measurable. For example, the level set $\{x: j(x)=2\}$ is measurable because it equals $\left\{x: M_{d}^{\sharp} \neq O_{Q_{1}}\right.$ and $\left.M_{d}^{\sharp}=O_{Q_{2}}\right\}$.

Now define the family of cubes by $Q(x)=Q_{j(x)}$. Let

$$
\kappa(x, y)=\frac{\overline{f(y)-f_{Q(x)}}}{\left|f(y)-f_{Q(x)}\right|}, \quad x, y \in \mathbb{R}^{n},
$$

with the convention that if the denominator equals 0 then $\kappa(x, y)=0$. Clearly $|\kappa| \leq 1$, and the representation (2) holds.

For the measurability claims in the proposition, the first observation is that the set

$$
P \stackrel{\text { def }}{=}\{(x, y): y \in Q(x)\}=\bigcup_{k=1}^{\infty}\left(\{x: j(x)=k\} \times Q_{k}\right)
$$

can be expressed as a union of measurable products. Some care is needed to prove measurability of $x \mapsto f_{Q(x)}$ and hence measurability of $\kappa$, as we now explain. Start with

$$
f_{Q(x)}=\frac{1}{|Q(x)|} \int_{\mathbb{R}^{n}} 1_{P}(x, y) f(y) d y .
$$

Observe $|Q(x)|=\int_{\mathbb{R}^{n}} 1_{P}(x, y) d y$, which is a measurable function of $x$ by Tonelli's theorem since $1_{P}$ is a nonnegative jointly measurable function. Next, for any dyadic cube $\widetilde{Q}$ we have

$$
\int_{\widetilde{Q}} \int_{\mathbb{R}^{n}} 1_{P}(x, y)|f(y)| d y d x \leq \int_{\widetilde{Q}}|Q(x)|^{1-1 / p}\|f\|_{L^{p}} d x<\infty
$$

by Hölder's inequality and the locally uniform upper bound on $|Q(x)|$ in Lemma 6 . Hence the integrand $1_{P}(x, y) f(y)$ is a jointly integrable function of $(x, y) \in \widetilde{Q} \times \mathbb{R}^{n}$, and so $\int_{\mathbb{R}^{n}} 1_{P}(x, y) f(y) d y$ is a measurable function of $x \in \widetilde{Q}$ by Fubini's theorem. Thus by (3) we see that $f_{Q(x)}$ is measurable for $x \in \widetilde{Q}$. Since the cube $\widetilde{Q}$ was arbitrary, measurability follows on all of $\mathbb{R}^{n}$. 


\section{Norm inequalities for the dyadic $\#$-maximal operator}

Measure and norm estimates will be needed on the maximal operators.

LEMMA 8 (Dyadic good lambda). If $f$ is locally integrable then

$$
\left|\left\{x \in \mathbb{R}^{n}: M_{d} f(x)>\alpha(1+\beta), M_{d}^{\sharp} f(x) \leq \alpha \gamma\right\}\right| \leq 2^{n} \frac{\gamma}{\beta}\left|\left\{x \in \mathbb{R}^{n}: M_{d} f(x)>\alpha\right\}\right|
$$

whenever $\alpha, \beta, \gamma>0$.

Proof. See Stein [8, page 153], and make there the substitutions $\alpha \mapsto \alpha(1+\beta), b \mapsto$ $1 /(1+\beta)$ and $c \mapsto \gamma /(1+\beta)$.

Proposition 9 (Maximal norm dominated by $\sharp$-maximal norm). If $1 \leq q<p<\infty$ and $f \in L^{q}$, then

$$
\|f\|_{L^{p}} \leq\left\|M_{d} f\right\|_{L^{p}} \leq 2^{n} K(p)\left\|M_{d}^{\sharp} f\right\|_{L^{p}},
$$

where $K(p)$ was defined in the Introduction.

The previous best constant in the literature is $2^{n+2+1 / p} 2^{p}$, appearing in the text of Stein [8, pages 153-154] and also treated by Grafakos [5, Theorem 7.4.5]. (For the nondyadic maximal and $\sharp$-maximal operators, Fefferman and Stein got a larger constant, of order $2^{(n+1) p}$ [3, Theorem 5].)

Our constant $2^{n} K(p)$ grows only linearly with $p$. The improvement comes from optimizing our choice of parameters when using the good lambda inequality. For example, Stein chose $\beta=1$, whereas we will identify an optimal $\beta$ that depends on $p$.

Proof. For the first inequality in Proposition 9, recall $|f| \leq M_{d} f$ a.e. by the Lebesgue differentiation theorem.

We use the good lambda inequality (4) to prove the second inequality. Let

$$
I(A, f)=\int_{0}^{A} p \alpha^{p-1}\left|\left\{x \in \mathbb{R}^{n}:|f(x)|>\alpha\right\}\right| d \alpha
$$

so that $\|f\|_{L^{p}}^{p}=\lim _{A \rightarrow \infty} I(A, f)$. Note that $I\left(A, M_{d} f\right)$ is finite for each $A>0$, using here that $p>q$ and calling on the weak $\left(L^{q}, L^{q}\right)$ estimate on the dyadic maximal operator. Let

$$
\delta=1+\beta,
$$

so that $\delta>1$. Changing variable with $\alpha \mapsto \alpha \delta$ in the integral shows that

$$
\begin{aligned}
I\left(A, M_{d} f\right) & =\delta^{p} \int_{0}^{A / \delta} p \alpha^{p-1}\left|\left\{x \in \mathbb{R}^{n}: M_{d} f(x)>\alpha \delta\right\}\right| d \alpha \\
= & \delta^{p} \int_{0}^{A / \delta} p \alpha^{p-1}\left|\left\{x \in \mathbb{R}^{n}: M_{d} f(x)>\alpha \delta, M_{d}^{\sharp} f(x) \leq \alpha \gamma\right\}\right| d \alpha \\
& \quad+\delta^{p} \int_{0}^{A / \delta} p \alpha^{p-1}\left|\left\{x \in \mathbb{R}^{n}: M_{d} f(x)>\alpha \delta, M_{d}^{\sharp} f(x)>\alpha \gamma\right\}\right| d \alpha
\end{aligned}
$$




$$
\begin{aligned}
& \leq 2^{n} \gamma \beta^{-1} \delta^{p} \int_{0}^{A / \delta} p \alpha^{p-1}\left|\left\{x \in \mathbb{R}^{n}: M_{d} f(x)>\alpha\right\}\right| d \alpha \quad \text { by Lemma } 8 \\
& \quad+\delta^{p} \int_{0}^{A / \delta} p \alpha^{p-1}\left|\left\{x \in \mathbb{R}^{n}: M_{d}^{\sharp} f(x)>\alpha \gamma\right\}\right| d \alpha \\
& \leq 2^{n} \gamma \beta^{-1} \delta^{p} I\left(A, M_{d} f\right)+\delta^{p} \gamma^{-p} I\left(A \gamma / \delta, M_{d}^{\sharp} f\right) .
\end{aligned}
$$

If $\beta$ and $\gamma$ are restricted to satisfy $2^{n} \gamma \beta^{-1}(1+\beta)^{p}<1$, then by letting $A \rightarrow \infty$ in the preceding inequality and recalling that $\delta=1+\beta$ we see

$$
\left\|M_{d} f\right\|_{L^{p}}^{p} \leq \frac{(1+\beta)^{p} \gamma^{-p}}{1-2^{n} \gamma \beta^{-1}(1+\beta)^{p}}\left\|M_{d}^{\sharp} f\right\|_{L^{p}}^{p} .
$$

Rescaling the parameter $\gamma$ to $2^{-n} \beta(1+\beta)^{-p} \gamma$ gives that

$$
\left\|M_{d} f\right\|_{L^{p}}^{p} \leq \frac{\left(2^{n}(1+\beta)^{p+1} / \beta\right)^{p}}{\gamma^{p}(1-\gamma)}\left\|M_{d}^{\sharp} f\right\|_{L^{p}}^{p},
$$

where the earlier restriction on $\beta$ and $\gamma$ rescales to say simply that $\gamma<1$. We minimize the numerator by choosing $\beta=1 / p$ and maximize the denominator by choosing $\gamma=p /(p+1)<1$. With these choices we evaluate the constants and take the $p$ th root to find that

$$
\left\|M_{d} f\right\|_{L^{p}} \leq 2^{n}(1+1 / p)^{p+2}(p+1)^{1 / p} p\left\|M_{d}^{\sharp} f\right\|_{L^{p}}=2^{n} K(p)\left\|M_{d}^{\sharp} f\right\|_{L^{p}} .
$$

This estimate concludes the proof.

\section{Proof of Theorem 1}

We modify the proof of Fefferman-Stein [3, pages 156-157] by using dyadic cubes when defining both the $\sharp$-maximal operator and their $U$-operator. Note that their $U f$ is the right side of (2) applied to $T f$ instead of $f$, for an arbitrary family of cubes $\{Q(\cdot)\}$ and an arbitrary kernel $\kappa$ with $|\kappa| \leq 1$.

The last part of Fefferman and Stein's argument relies on representing $M^{\sharp}(T f)$ as the supremum of $U f$ over all families of cubes and over all kernels. For our dyadic version of the argument, it is easy to show that equality is attained in this supremum, assuming $f$ is simple. Indeed, $f \in L^{2}$ and hence $T f \in L^{2}$, and so we may apply Proposition 7 to $T f$ with $p=2$ to obtain a measurable choice of $Q(\cdot)$ and $\kappa$ such that $M_{d}^{\sharp}(T f)=U f$ as desired.

From these dyadic modifications of Fefferman and Stein's complex interpolation arguments we obtain $L^{p}$-boundedness of the dyadic $\sharp$-maximal operator composed with $T$; more precisely, we get for all simple $f$ that

$$
\left\|M_{d}^{\sharp}(T f)\right\|_{L^{p}} \leq A(p)\|f\|_{L^{p}}, \quad 2<p<\infty,
$$


where the constant is

$$
A(p)=\left(4\|T\|_{L^{2} \rightarrow L^{2}}\right)^{2 / p}\|T\|_{L^{\infty} \rightarrow d B M O}^{1-2 / p}
$$

The factor of 4 arises in the derivation of $A(p)$ because $M_{d}^{\sharp} f \leq 2 M_{d} f$ by the triangle inequality and $\left\|M_{d}\right\|_{L^{2} \rightarrow L^{2}} \leq 2$.

To obtain $L^{p}$-boundedness of $T$ itself acting on simple functions, we argue that

$$
\begin{aligned}
\|T f\|_{L^{p}} \leq & \left\|M_{d}(T f)\right\|_{L^{p}} \\
\leq & 2^{n} K(p)\left\|M_{d}^{\sharp}(T f)\right\|_{L^{p}} \\
& \quad \text { by Proposition } 9 \text { applied to } T f \text { with } q=2 \\
\leq & 2^{n} K(p) A(p)\|f\|_{L^{p}}
\end{aligned}
$$

by (5) above. Finally we extend the inequality to all $f \in L^{p}$, by density.

Note. Compared with Fefferman and Stein's proof, and others in the literature, the improvement in the constant in our theorem comes mostly from Proposition 9, which optimally applies the good lambda inequality.

Proof of Corollary 3. The adjoint $T^{*}$ is strong type $\left(L^{2}, L^{2}\right)$, and strong type $\left(L^{\infty}, B M O\right)$ with

$$
\left\|T^{*}\right\|_{L^{\infty} \rightarrow\left(H^{1}\right)^{*}}=\|T\|_{H^{1} \rightarrow L^{1}} .
$$

The norm on the left uses the dual norm on $\left(H^{1}\right)^{*}$, which does not equal the $B M O$ norm. The norms are equivalent, fortunately, and Lemma 10 below implies that

$$
\left\|T^{*}\right\|_{L^{\infty} \rightarrow B M O} \leq 2\left\|T^{*}\right\|_{L^{\infty} \rightarrow\left(H^{1}\right)^{*}}=2\|T\|_{H^{1} \rightarrow L^{1}} .
$$

Then Theorem 1 together with inequality (6) gives that

$$
\left\|T^{*}\right\|_{L^{p^{\prime}} \rightarrow L^{p^{\prime}}} \leq 2^{n+4 / p^{\prime}} K\left(p^{\prime}\right)\|T\|_{L^{2} \rightarrow L^{2}}^{2 / p^{\prime}}\left(2\|T\|_{H^{1} \rightarrow L^{1}}\right)^{1-2 / p^{\prime}},
$$

where $p^{\prime}=p /(p-1)$ is the dual exponent to $p$. The corollary now follows by duality.

Equivalence of the $H^{1}$-dual norm and the $B M O$ norm, as stated in the next lemma, is well known. We provide a proof in order to get explicit constants independent of the dimension. The proof is based on the treatment by Coifman and Weiss [2, pages 632-633].

Lemma 10 (Norm equivalence on BMO). If L is a linear functional in $\left(H^{1}\right)^{*}$ that acts by integration against the function $b \in B M O$, then

$$
\|L\|_{\left(H^{1}\right)^{*}} \leq\|b\|_{B M O} \leq 2\|L\|_{\left(H^{1}\right)^{*}} .
$$

Proof. If $f \in H^{1}$ has atomic decomposition $f=\sum_{k} c_{k} a_{k}$ (where the $a_{k}$ are atoms with mean zero, supported on cubes $Q_{k}$ and with $\left.\left\|a_{k}\right\|_{L^{\infty}} \leq\left|Q_{k}\right|^{-1}\right)$, then

$$
L f=\sum_{k} c_{k} \int_{Q_{k}} a_{k} b d x=\sum_{k} c_{k} \int_{Q_{k}} a_{k}\left(b-b_{Q_{k}}\right) d x .
$$


Hence $|L f| \leq \sum_{k}\left|c_{k}\left\|\left.Q_{k}\right|^{-1} \int_{Q_{k}}\left|b-b_{Q_{k}}\right| d x \leq\right\| b \|_{B M O} \sum_{k}\right| c_{k} \mid$. Taking the infimum over all atomic decompositions gives that $|L f| \leq\|b\|_{B M O}\|f\|_{H^{1}}$ and so $\|L\|_{\left(H^{1}\right)^{*}} \leq\|b\|_{B M O}$.

For the other direction, fix a cube $Q$ and choose $f \in L^{\infty}(Q)$ with $|f|=1$ a.e. such that $\left(b-b_{Q}\right) f=\left|b-b_{Q}\right|$. Define a new function on $Q$ by $a=\left(f-f_{Q}\right) / 2|Q|$, and notice this function $a$ is an atom because it has mean zero and $\|a\|_{L^{\infty}} \leq 1$. Then

$$
\begin{aligned}
L a=\int_{Q} a b d x & =\frac{1}{2|Q|} \int_{Q}\left(f-f_{Q}\right) b d x \\
& =\frac{1}{2|Q|} \int_{Q} f\left(b-b_{Q}\right) d x=\frac{1}{2|Q|} \int_{Q}\left|b-b_{Q}\right| d x .
\end{aligned}
$$

Therefore,

$$
\frac{1}{|Q|} \int_{Q}\left|b-b_{Q}\right| d x=2 L a \leq 2\|L\|_{\left(H^{1}\right)^{*}},
$$

since $\|a\|_{H^{1}} \leq 1$ by definition of the atomic norm. Taking the supremum over all cubes now shows that $\|b\|_{B M O} \leq 2\|L\|_{\left(H^{1}\right)^{*}}$.

\section{Acknowledgements}

Laugesen thanks the Department of Mathematics and Statistics at the University of Canterbury, New Zealand, for hosting him during the initial stages of this research. Bui thanks the Department of Mathematics at the University of Illinois, USA, for hospitality during the completion of the work. We are grateful to the referee for numerous suggestions that improved the exposition of the paper.

\section{References}

[1] H.-Q. Bui and R. S. Laugesen, 'Wavelet frame bijectivity on Lebesgue and Hardy spaces', J. Fourier Anal. Appl. 19 (2013), 376-409.

[2] R. R. Coifman and G. Weiss, 'Extensions of Hardy spaces and their use in analysis', Bull. Amer. Math. Soc. 83(4) (1977), 569-645.

[3] C. Fefferman and E. M. Stein, ' $H^{p}$ spaces of several variables', Acta Math. 129(3-4) (1972), 137-193.

[4] J. B. Garnett and P. W. Jones, 'BMO from dyadic BMO', Pacific J. Math. 99(2) (1982), 351-371.

[5] L. Grafakos, Modern Fourier Analysis, 2nd edn, Graduate Texts in Mathematics, 250 (Springer, New York, 2009).

[6] T. Iwaniec and G. Martin, Geometric Function Theory and Nonlinear Analysis, Oxford Mathematical Monographs (The Clarendon Press, Oxford University Press, New York, 2001).

[7] S. K. Pichorides, 'On the best values of the constants in the theorems of M. Riesz, Zygmund and Kolmogorov'. Collection of articles honoring the completion by Antoni Zygmund of 50 years of scientific activity, II. Studia Math. 44 (1972), 165-179.

[8] E. M. Stein, with the assistance of Timothy S. Murphy. Harmonic Analysis: Real-variable Methods, Orthogonality, and Oscillatory Integrals, Princeton Mathematical Series, 43. Monographs in Harmonic Analysis, III (Princeton University Press, Princeton, NJ, 1993). 
H.-Q. BUI, Department of Mathematics, University of Canterbury,

Christchurch 8020, New Zealand

e-mail: Huy-Qui.Bui@ canterbury.ac.nz

R. S. LAUGESEN, Department of Mathematics, University of Illinois,

Urbana, IL 61801, USA

e-mail: Laugesen@illinois.edu 